\title{
Novel Raman-tagged sphingomyelin that closely mimics original raft-forming behavior
}

\author{
Jin Cui ${ }^{\text {a,b,c }}$, Shigeru Matsuoka ${ }^{\text {a,b,c }}$, Masanao Kinoshita ${ }^{\text {a,b,c } \dagger}$, Nobuaki Matsumori ${ }^{\text {a, } \dagger}$, Fuminori Sato ${ }^{\text {b,c }}$, Michio \\ Murata $^{\text {a,b,c,** }}$, Jun Ando ${ }^{\text {d,e,f,g }}$, Hiroyuki Yamakoshi ${ }^{\text {d,e }}$, Kosuke Dodo ${ }^{\text {d,e,f }}$, Mikiko Sodeoka ${ }^{\text {d,e,f }}$ \\ ${ }^{a}$ Department of Chemistry, Osaka University, 1-1 Machikaneyama, Toyonaka, Osaka 560-0043, Japan \\ ${ }^{b}$ JST, ERATO, Lipid Active Structure Project, Osaka University, 1-1 Machikaneyama, Toyonaka, Osaka 560-0043, Japan \\ ${ }^{c}$ Project Research Centre for Fundamental Science, Osaka University, 1-1 Machikaneyama, Toyonaka, Osaka 560-0043, Japan \\ d JST, ERATO, Sodeoka Live Cell Chemistry Project, 2-1 Hirosawa, Wako, Saitama 351-0198, Japan \\ e Synthetic Organic Chemistry Laboratory, RIKEN, 2-1 Hirosawa, Wako, Saitama 351-0198, Japan \\ ${ }^{f}$ CREST, JST, 2-1 Hirosawa, Wako, Saitama 351-0198, Japan \\ ${ }^{g}$ Department of Applied Physics, Osaka University, 2-1 Yamadaoka, Suita, Osaka, 565-0871, Japan \\ ${ }^{\dagger}$ Present address: Department of Chemistry, Graduate School of Sciences, Kyushu University, Higashi-ku, Fukuoka 812-8581, \\ Japan \\ * Corresponding author. Tel./fax: +81 66850 5774. E-mail address: murata@chem.sci.osaka-u.ac.jp
}

Keywords: sphingomyelin, lipid rafts, Raman imaing, solid-state ${ }^{2} \mathrm{H}$ NMR, phase distribution

\begin{abstract}
Three Raman probes of sphingomyelin (SM) were synthesized and evaluated for their applicability to imaging experiments. One probe containing a hydroxymethyl-1,3-butadiyne moiety in the polar head group showed strong scattering. The solid-state ${ }^{2} \mathrm{H}$ NMR spectra of this probe in oriented bilayer membrane revealed excellent compatibility with natural SM in phase behavior since the probe undergoes phase separation to form raft-like liquid ordered $\left(\mathrm{L}_{\mathrm{o}}\right)$ domains in the raft-mimicking mixed bilayers.
\end{abstract}

\section{Introduction}

Lipid rafts show a similar character to the lipid ordered $\left(\mathrm{L}_{\mathrm{o}}\right)$ phase in artificial membranes, which are often represented by microdomains enriched with sphingolipids (SM) and cholesterol (chol). The physicochemical properties of the specific lipids are believed to be important for fulfilling the raft functions such as cellular signal transduction. ${ }^{1}$ To gain better insight into the role of lipid rafts in these processes, a direct optical visualization of such domains is essential. However, the task in raft imaging has not been truly achieved due to the lack of a suitable raft-specific probe. Fluorescent microscopy, which is commonly used for observing molecular localization, requires doping of the membranes with fluorescently labeled lipids. However, the probes attached with a relatively large fluorophore possess physical properties that are somewhat different from those of small lipid molecules, and often alter the membrane properties. ${ }^{2}$ In model membranes, the chemical modification of a lipid molecule with a fluorescent moiety usually results in an inconsistent, or even completely opposite, distribution tendency in the membrane phases. $^{3}$

In contrast, Raman-active moieties are considered to have less influence on the membrane properties due to their small size. The Raman image can be generated by measuring a specific molecular vibration to map the Raman-tagged molecules. ${ }^{4}$ Functional groups such as diyne, azide, deuterium, and nitrile groups give rise to Raman scattering bands in the cellular silent region $\left(1800-2800 \mathrm{~cm}^{-1}\right)$, where most endogenous biomolecules do not exhibit a signal. ${ }^{5}$ Alkyne-tagged coenzyme Q (AltQ) and 5-ethynyl-2'-deoxyuridine (EdU) analogs have been recently used for imaging their distribution in living HeLa cells. ${ }^{5}$ The most significant features of this tag are its suitable wavenumber of the Raman band and strong scattering intensity, which allow high-contrast imaging. Deuterium is expected to be another promising candidate due to its small size and good physical compatibility. Although the Raman intensity derived from the C-D bond is relatively week, multiple-deuterated methylene or methyl groups may generate relatively strong Raman signals. Thus, we deduced that these tags could be further applied in raft-specific lipids to mark raft-like domains. 
In this study, we designed and synthesized Raman-tagged SMs at the polar head, and measured their Raman spectra in a monolayer form. For promising candidates, we further evaluated their membrane behavior using ${ }^{2} \mathrm{H}$ solid state NMR with oriented bilayer membranes. Probes $\mathbf{1}$ and $\mathbf{2}$ possess terminal alkyne and hydroxymethyl-1,3-butadiyne groups, respectively; Probe $\mathbf{3}$ is labeled with nine deuterium atoms at the trimethyl ammonium moiety (Figure 1), which encompasses the largest number of chemically equivalent $\mathrm{C}-\mathrm{D}$ bonds in the polar head of SM. All of the probes possess a stearoyl (C18:0) moiety since stearic acid is one of the common acyl groups in SMs, particularly in those of bovine brain. ${ }^{6}$

In order to observe domain formation by Raman microscopy, previous studies have adopted acyl-labeled Raman probes such as DPPC- $d_{62}$ or DSPC- $d_{70}$, in which the glycerophospholipid was substituted with perdeuterated fatty acids. $^{7}$ Although the acyl-deuterated probes are believed to have excellent compatibility with the natural lipids, the hydrocarbon packing in membrane are slightly different between highly deuterated and non-deuterated acyl chains. $^{7 \mathrm{a}, 8}$ The lipid rafts are best characterized by the highly packed acyl chains, as is the case with the $\mathrm{L}_{\mathrm{o}}$ phase in artificial membranes. A small but significant perturbation in the acyl chain behavior caused by deuteration may hamper us from elucidating the precise phase properties of the original lipid. In contrast, since the polar head of phospholipids is relatively loosely packed even in the $\mathrm{L}_{\mathrm{o}}$-phase, this portion is occasionally more suitable for chemical modifications. Thus, for investigating the atomistic mechanism underlying formation of the raft-mimicking $\mathrm{L}_{\mathrm{o}}$ phase, besides acyl chain-tagged Raman probes, those with a small tag on the head group can be complimentarily used in the imaging of membrane domains.

\section{Results and discussion}

Alkyne-SM 1 was first synthesized from Boc-L-serine through a protected sphingosine using a partially modified procedure from those in previous reports (Scheme 1). ${ }^{9,10}$ Initially, we attempted to introduce the conjugated diyne moiety using 3-bromoprop-2-yn-1-ol as the coupling partner under the classical Cadiot-Chodkiewicz coupling conditions (copper(I) chloride, $5 \mathrm{~mol} \%$; hydroxylamine, $30 \mathrm{~mol} \%$; $i$ - $\mathrm{PrNH}_{2}$ in aqueous methanol), ${ }^{11}$ as reported separately. ${ }^{12}$ Unfortunately, it was difficult to drive the coupling to completion and only a modest conversion was achieved, as judged by ${ }^{1} \mathrm{H}$ NMR. Furthermore, the remaining alkyne $\mathbf{1}$ was hardly separable from compound $\mathbf{2}$ due to their similar polarities. Lei's group reported a mild $\mathrm{Ni} / \mathrm{Cu}$-cocatalyzed oxidative coupling reaction, by which the $\mathrm{C}_{\mathrm{sp}}-\mathrm{C}_{\mathrm{sp}}$ bond formation was performed in favor of the heterocoupled product over the homocoupled products by increasing the molar ratio of the two terminal alkynes. ${ }^{13}$ Based on their conditions, the coupling of $\mathbf{1}$ with a large excess of propargyl alcohol enabled selective conversion to $\mathbf{2}$, which was easily separated by flash chromatography, although the isolated yield was modest (40\%).

The synthetic route for SM- $d_{9} 3$ is shown in Scheme 2. Substrate 6, which was synthesized from Boc-L-serine ${ }^{9}$, was treated with $\mathrm{N}\left(\mathrm{CD}_{3}\right)_{3}$ in $\mathrm{MeOH}$ furnished 7 in a modest yield. Boc deprotection of 7 by TFA, followed by acylation with $p$-nitrophenyl stearate, produced the desired compound $\mathbf{3}$.

The deuterated side chains of $\mathbf{4}$ and $\mathbf{5}$ were synthesized by adopting a more efficient route including a copper-catalyzed Kumada-Corriu coupling ${ }^{14}$ as the key step compared to previously reported method ${ }^{15}$. (Scheme 3 ) Protection of the primary alcohol group in $\mathbf{8}$ afforded compound $\mathbf{9}$. Reduction of the ester with $\mathrm{LiAlD}_{4}$, followed by tosylation, provided deuterated intermediate 11. The tosylated precursor has been considered as an efficient coupling partner for the $\mathrm{C}_{\mathrm{sp} 3}-\mathrm{C}_{\mathrm{sp} 3}$ coupling. ${ }^{16}$ Compound 11 was subsequently coupled with a Grignard reagent to afford 12 in excellent yield (91\%). Removal of TBDPS, followed by Jones' oxidation, led to deuterated 14, which was then converted into the corresponding active ester $\mathbf{1 5}$ by a condensation reaction with $p$-nitrophenol. Using the same synthetic method as for compound $\mathbf{1}$ and $\mathbf{2}$, the deuterated samples $\mathbf{4}$ and 5 were synthesized. ${ }^{17}$

With these Raman-tagged SMs in hand, we first compared the relative Raman shift and intensity using their 
monolayer systems prepared by the Langmuir-Blodgett (LB) technique. As shown in Figure 2, the signals from Raman tags, such as diynes, alkynes, and C-D bonds, are observed in the cellular Raman-silent region, where no Raman band occurs from non-labeled SM, 1,2-dioleoyl-sn-glycero-3-phosphocholine (DOPC) or chol; these three lipids are the constituents of raft-model membranes. The triple bond in alkyne-SM 1 shows a single Raman peak at $2132 \mathrm{~cm}^{-1}$. The conjugated triple bonds in diyne-SM 2 show a significantly enhanced intensity and peak shifts to longer wavenumber at $2263 \mathrm{~cm}^{-1}$, leading to an approximately five-fold greater peak height than that of the alkyne in $\mathbf{1}$; the Raman imaging of the distribution of $\mathbf{2}$ in phase-separated membranes has been published. ${ }^{12}$ In the case of SM- $d_{9}$ 3, the observed peaks can be assigned to symmetric $\mathrm{CD}_{3}$ stretching at $2184 \mathrm{~cm}^{-1}$, and Fermi resonance multiplets at $2132 \mathrm{~cm}^{-1}$ and $2081 \mathrm{~cm}^{-1}$ with overtone or combination tone of $\mathrm{CD}_{3}$ deformation. ${ }^{18}$ A peak due to asymmetric $\mathrm{CD}_{3}$ stretching at $\sim 2276 \mathrm{~cm}^{-1}$ was unnoticeable owing to the overlap with rotation-vibrational Raman modes of nitrogen in air around $2332 \mathrm{~cm}^{-1}$. The apparent Raman intensity of the deuterated methyl groups is lower than that of alkyne $\mathbf{1}$. Since compound $\mathbf{3}$, which could be the best candidate in terms of compatibility in phase behavior, did not give rise to intense Raman bands, we concentrated on evaluating the compatibility of $\mathbf{1}$ and 2 with natural SM using solid-state ${ }^{2} \mathrm{H}$ NMR.

Using ${ }^{2} \mathrm{H}$ NMR of $3 \alpha$-deuterated chol (chol- $d$ ), ${ }^{19}$ we could quantify the molecular ordering of chol in the host lipid bilayers and quantitatively characterize the physicochemical properties of the mixed lipid membranes. ${ }^{20} \mathrm{We}$ first measured the signals of chol- $d$ in an oriented binary system composed of equimolar proportions of chol- $d$ and DOPC, a typical unsaturated lipid that formed the liquid disordered $\left(\mathrm{L}_{\mathrm{d}}\right)$ phase. As shown in Figure $3 \mathbf{a}$, the ${ }^{2} \mathrm{H}$ NMR spectra of chol- $d$ provided a sharp quadrupolar doublet $(45.3 \mathrm{kHz})$, which was assigned to chol- $d$ embedded in the DOPC bilayers. ${ }^{21}$ Among artificial membranes, ternary SM/chol/DOPC bilayers are often used as a model to mimic raft-like domains. In this system (Figure 3b), a second set of more ordered peaks appeared, indicating that the raft-like liquid ordered $\left(\mathrm{L}_{\mathrm{o}}\right)$ domains were formed. The inner shoulders correspond to the DOPC-rich $\mathrm{L}_{\mathrm{d}}$ phases as indicated by the same splitting width as that of the DOPC-chol membrane in Figure 3a. The major fraction of chol- $d$ was partitioned to the $\mathrm{L}_{\mathrm{o}}$ phases, as shown by the higher intensity of the outer quadrupole splitting. The $\mathrm{L}_{\mathrm{o}} / \mathrm{L}_{\mathrm{d}}$ immiscibility was also observed in the 1/chol-d/DOPC (1/1/1 mol) and 2/chol-d/DOPC (1/1/1 mol) mixtures (Figure 3c and 3d). Similarly, the preference of chol by $\mathbf{1}$ and $\mathbf{2}$ was revealed by the presence of the larger outer doublets. The intensity of the inner peak of $\mathbf{1}$ is comparable to that of the SM mixture; the inner doublet peak of the $\mathbf{2}$-containing mixture was slightly higher than that of the SM and $\mathbf{1}$ ternary mixtures. Since the quadrupolar splitting magnitude $\Delta v_{\mathrm{Q}}$ is largely dependent on the ordering of phospholipid/chol mixed domains under the present conditions, ${ }^{21,23}$ the observed $\Delta v_{\mathrm{Q}}$ values, which are usually expressed in terms of the molecular order parameter $\left(S_{\mathrm{CD}}\right)$, largely represents the wobbling motion of the rigid skeleton of chol in the $\mathrm{L}_{\mathrm{o}}$ and $\mathrm{L}_{\mathrm{d}}$ phases. For each phase, the almost identical $S_{\mathrm{CD}}$ values are observed for the SM, 1, and 2 mixtures, indicating that the membrane rigidity and/or fluidity for the $\mathbf{1}$ and $\mathbf{2}$ mixtures are very similar to those of SM.

To further assess their similarity to SM in phase behaviour, we compared the lateral distribution and ordering of deuterated alkyne-SM 4 and diyne-SM 5 with deuterated SM (SM- $\left.d_{2}\right)$. Herein, site-selectively ${ }^{2} \mathrm{H}$-labeled compounds were used to avoid overlapping of quadrupole doublet signals found in perdeuterated acyl chains ${ }^{24}$, which can alter the physical properties of lipid bilayers as well ${ }^{25}$. Our previous study indicated that chol enhances the order of the $\mathrm{C} 10$ position more effectively than other positions in the acyl chains of SM, ${ }^{15}$ implying that the ${ }^{2} \mathrm{H}$ NMR spectrum should show clearly separated signals for the $\mathrm{L}_{\mathrm{o}}$ and $\mathrm{L}_{\mathrm{d}}$ phases. However, the clear phase separation of $\mathbf{5}$ is hardly observed in liposome preparation. ${ }^{12}$ Thus, we adopted magnetically oriented membrane as a model to observe the minor signals due to $\mathbf{4}$ and 5 partitioned to the $\mathrm{L}_{\mathrm{d}}$ phase. Figure 4 shows the ${ }^{2} \mathrm{H}$ NMR spectra of $d_{2}$-SM/chol/DOPC (1/1/1 mol), 4/chol/DOPC (1/1/1 mol), and 5/chol/DOPC (1/1/1 mol). Two pairs of resolved doublets are displayed for $\mathrm{SM}-d_{2} / \mathrm{chol} / \mathrm{DOPC}$, indicating the presence of two immiscible liquid phases 
with different lipid components, which confirmed the observed $\mathrm{L}_{\mathrm{o}}$ formation in the SM/chol-d/DOPC mixtures (Figure $3 \mathbf{b})$. A large majority of SM- $d_{2}$ is found in the $\mathrm{L}_{\mathrm{o}}$ phases with a quadrupolar splitting of $55.3 \mathrm{kHz}\left(S_{\mathrm{CD}}=\right.$ $0.44)$, while a small fraction pertains to the $\mathrm{L}_{\mathrm{d}}$ phases, as characterized by a splitting at $34.1 \mathrm{kHz}\left(S_{\mathrm{CD}}=0.27\right)$.

A comparison of the spectra of $\mathbf{4}$ and $\mathbf{5}$ with that of SM- $d_{2}$ shows a high degree of conformity in phase redistribution. Excellent preferences of $\mathbf{4}$ and $\mathbf{5}$ for the $\mathrm{L}_{\mathrm{o}}$ phase were observed (Figure $4 \mathbf{b}$ and $4 \mathbf{c}$ ), although the intensity of the inner small peak of $\mathbf{5}$ is slightly larger than those of the other two membranes. The quadrupolar splitting magnitudes of 4 and 5 in the $\mathrm{L}_{\mathrm{o}}$ phase are $\Delta v_{\mathrm{Q}}=55.2 \mathrm{kHz}\left(S_{\mathrm{CD}}, 0.44\right)$, and $\Delta v_{\mathrm{Q}}=55.4 \mathrm{kHz}\left(S_{\mathrm{CD}}, 0.44\right)$, respectively; these $S_{\mathrm{CD}}$ values of $\mathbf{4}$ and $\mathbf{5}$, which are almost identical with that of SM- $d_{2}$, are close to the maximum value, 0.5 , indicating that these ternary mixtures show a typical characteristic of the $\mathrm{L}_{\mathrm{o}}$ phases. ${ }^{26}$ The results revealed that the molecular orientation and mobility in the raft-like $\mathrm{L}_{\mathrm{o}}$ domains are minimally affected by the alkyne and diyne head groups. As shown in Table 2, the analogues showed increased quadrupolar splitting of the inner peaks compared with that of SM- $d_{2}$. In the $\mathrm{L}_{\mathrm{d}}$ phases, the $\Delta v_{\mathrm{Q}} / S_{\mathrm{CD}}$ values of SM- $d_{2}, 4$ and 5 are 34.1 $\mathrm{kHz} / 0.27,38.3 \mathrm{kHz} / 0.30$ and $41.0 \mathrm{kHz} / 0.33$, respectively. The increase in order is accompanied by an increase of head group size. It is worth noting that a similar tendency is observed with the intensity of the inner peak of chol (from Figure $3 \mathbf{b}$ to $3 \mathbf{d}$ ). Thus, the ordering effect of chol may be slightly increased in the $\mathrm{L}_{\mathrm{d}}$ phases of $\mathbf{4}$ and $\mathbf{5}$ due to the higher chol content in their $\mathrm{L}_{\mathrm{d}}$ phases.

In this study we developed the Raman probe of SM that closely mimics the original raft lipid. However, the probe cannot be directly applied to Raman imaging of biological membranes largely due to the detection limit of the instrument. Additional improvements in the performance of Raman microscopy are keenly awaited to attain higher speed and sensitivity necessary for imaging living cells.

\section{Conclusion}

It is taken for granted that some of the Raman-active moieties such as C-D bonds are small enough and not obviously affect the physical properties of biomolecules. However, there is a small but significant disturbance of the hydrophobic lipid interactions as previously reported for side-chain perdeuterated lipids. ${ }^{7,8}$ Thus, the potential perturbation in membrane, especially in raft-like domains, should be considered, and modification of the hydrophobic regions of lipids should be avoided or minimalized to accurately evaluate the domain formation of raft-specific lipids such as SM. In this study, we showed the rational design and preparation of three Raman-active SMs bearing alkyne, diyne and multiple C-D bonds on the polar head group. In a supported monolayer-membrane, they generated characteristic Raman peaks in the cellular silent region. Our current results indicate that diyne SM 2 is the best candidate for the high contrast observation of raft-like domains over a reasonable time frame. Firstly, it gives rise to a strong and sharp Raman peak as compared with alkyne SM 1 and SM- $d_{9}$ 3. Secondly, the ${ }^{2} \mathrm{H}$ NMR spectra demonstrate the similarity in raft-like domain formation between 2 and natural SM. Such features may enhance its versatility in combination with $\mathrm{L}_{\mathrm{d}}$ phase-selective fluorescent probes, which enables us to separately observe the $\mathrm{L}_{o}$ and $\mathrm{L}_{d}$ phases with a single membrane preparation. Further investigation of Raman imaging using the developed probe coupled with ${ }^{2} \mathrm{H}$ NMR spectra and fluorescence microscopy, will give new insights into the raft formation mechanism. 


\section{Experimental section}

\subsection{Synthesis}

See Supplementary data for details

\subsection{Materials}

Porcine brain SM and DOPC were purchased from Avanti Polar Lipids and Chol was purchased from Sigma-Aldrich. N-Stearoyl SM was extracted from brain SM and purified by HPLC (Cosmosil 5C18-AR-II column, $10 \times 150 \mathrm{~mm}$, Nacalai Tesque).

\subsection{Supported Monolayer Preparation.}

Monolayers of lipid mixtures were prepared on a computer-controlled Langmuir film balance (USI System) calibrated using stearic acid (Sigma Aldrich). The subphase, which consisted of distilled, freshly deionized water, was obtained using a Milli-Q System. The sample solution was prepared by mixing the appropriate amount of each lipid dissolved in chloroform $/ \mathrm{methanol}(4: 1 \mathrm{vol} / \mathrm{vol})$ in a microvial. A total of $30 \mu \mathrm{L}$ lipid solution $(1 \mathrm{mg} / \mathrm{mL})$ was spread onto the aqueous subphase $(100 \times 290 \mathrm{~mm})$ using a glass micropipette (Drummond Scientific Company). After an initial delay period of $10 \mathrm{~min}$ for evaporation of the organic solvent, the monolayers were compressed at a rate of $20 \mathrm{~mm}^{2} / \mathrm{s}$. The subphase and ambient temperatures were controlled at $25.0{ }^{\circ} \mathrm{C} \pm 0.1{ }^{\circ} \mathrm{C}$ and $25{ }^{\circ} \mathrm{C} \pm 2{ }^{\circ} \mathrm{C}$, respectively. A thin quartz plate was dipped horizontally into the water, followed by compression of the sample at $20 \mathrm{~mm}^{2} / \mathrm{s}$ to reach $\pi=12 \mathrm{mN} / \mathrm{m}$. After compression, the quartz substrate was extracted from the water at a rate of $0.1 \mathrm{~mm} / \mathrm{s}$, and quartz-supported monolayers were formed.

\subsection{Raman Measurements}

Raman spectra of lipid monolayers were obtained by Raman microscopy (Raman-11, Nanophoton) with a 532 $\mathrm{nm}$ excitation laser. The excitation laser was focused into a point at the sample with a water immersion objective lens (CFI Plan Apo IR 60XWI, Nikon). The exposure time was $6 \mathrm{~s}$ and the Raman measurement was performed 15 times per membrane. The averaged Raman spectra are displayed in Figure 2. The laser power at the sample was $340 \mathrm{~mW}$. Raman spectrum of the powder of SM- $d_{9}$ in Figure S1 was observed with same Raman microscopy. Exposure time was $10 \mathrm{sec}$. Laser power at the sample was $95 \mathrm{~mW}$.

\section{5. ${ }^{2} \mathrm{H}$ NMR Measurements of oriented membrane preparations}

For ${ }^{2} \mathrm{H}$ NMR measurements, $2 \mathrm{mg}$ of total lipids were dissolved in $n-\mathrm{PrOH} / \mathrm{MeOH} / \mathrm{CHCl}_{3}(70 \mu \mathrm{L}, 4: 1: 1 \mathrm{v} / \mathrm{v} / \mathrm{v})$. The lipid solution was applied to 14 micro cover glasses $(2 \times 5 \times 0.12-0.17 \mathrm{~mm}$, Matsunami Glass Ind., Ltd $)$, and then dried, first in air and thereafter under high vacuum for $20 \mathrm{~h}$. The slides were stacked in a culture dish and hydrated with $\mathrm{K}_{2} \mathrm{SO}_{4}$-saturated deuterium-depleted water at $40{ }^{\circ} \mathrm{C}$ for 3 days. The stacked plates were transferred into an open-ended NMR tube ( $3 \mathrm{~cm}$ long, $4 \mathrm{~mm}$ o.d.) which was sealed in advance with a plastic stopper and epoxy glue at one end. After rehydration overnight at $40{ }^{\circ} \mathrm{C}$, the sample was fixed with another plastic stopper and sealed with epoxy glue.

${ }^{2} \mathrm{H}$ NMR spectra were acquired on a Bruker Ultrashield $400 \mathrm{MHz}$ spectrometer with a Bruker $5 \mathrm{~mm}{ }^{2} \mathrm{H}$ static probe using a quadrupolar echo sequence. ${ }^{27}$ The $90^{\circ}$ pulse width was $5 \mu \mathrm{s}$, the interpulse delay was $24 \mu \mathrm{s}$, and recycle delay was $0.5 \mathrm{~s}$. The sweep width was $250 \mathrm{kHz}$, and the number of scans was 200000-400000.

\section{Acknowledgements}

We thank Mr. Tomokazu Yasuda for providing SM- $d_{2}$, and Dr. Yuichi Umegawa for his kind support for ${ }^{2} \mathrm{H}$ NMR measurements. We also thank Dr. Sebastien Lethu for helpful discussions. This work was supported by 
Japanese Society of Technology (JST) ERATO, Lipid Active Structure Project and by Grant-In-Aids for Scientific Research (A) (No. 25242073) from MEXT, Japan.

\section{Supplementary data}

Supplementary data associated with this article can be found, in the online version, at http://dx.doi.org./XXXXX.

\section{References and notes}

1. (a) Simons, K.; Ikonen, E. Nature 1997, 387, 569; (b) Simons, K.; Toomre, D. Nat. Rev. Mol. Cell Biol. 2000, 1, 31; (c) Brown, D. A.; Rose, J. K. Cell 1992. 68, 533; (d) Lingwood, D.; Simons, K. Science 2010, 327, 46.

2. Cruz, A.; Vazquez, V.; Velez, M.; Perez-Gil, J. Langmuir 2005, 21, 5349.

3. (a) Baumgart, T.; Hunt, G.; Farkas, E. R.; Webb, W. W.; Feigenson, G. W. Biochim. Biophys. Acta 2007, 1768, 2182; (b) Sezgin, E.; Levental, I.; Grzybek, M.; Schwarzmann, G.; Mueller, V.; Honigmann, A.; Belov, V. N.; Eggeling, C.; Coskun, U.; Simons, K.; Schwille, P. Biochim. Biophys. Acta 2012, 1818, 1777; (c) Klymchenko, A. S.; Kreder, R. Chem. Biol. 2014, 21, 97.

4. (a) Palonpon, A. F.; Sodeoka, M.; Fujita, K. Curr. Opin. Chem. Biol. 2013, 17, 708; (b) Yamakoshi, H.; Dodo, K.; Okada, M.; Ando, J.; Palonpon, A.; Fujita, K.; Kawata, S.; Sodeoka, M. J. Am. Chem. Soc. 2011, 133, 6102.

5. Yamakoshi, H.; Dodo, K.; Palonpon, A. F.; Ando, J.; Fujita, K.; Kawata, S.; Sodeoka, M. J. Am. Chem. Soc. 2012, 134, 20681.

6. Garner, A. E.; Smith, D. A.; Hooper, N. M. Mol. Mem. Biol. 2007, 24, 233.

7. (a) Mendelsohn, R.; Koch, C. C. Biochim. Biophys. Acta 1980, 598, 260; (b) Li, L.; Wang, H. F.; Cheng, J. X. Biophys. J. 2005, 89, 3480; (c) Potma, E. O.; Xie, X. S. Chem. Phys. Chem. 2005. 6, 77; (d) Opilik, L.; Bauer, T.; Schmid, T.; Stadler, J.; Zenobi, R. Phys. Chem. Chem. Phys. 2011. 13, 9978.

8. Guard-Friar, D.; Chen, C. H.; Engle, A. S. J. Phys. Chem. 1985, 89, 1810.

9. Yamamoto, T.; Hasegawa, H.; Hakogi, T.; Katsumura, S. Org. Lett. 2006, 8, 5569.

10. (a) Sandbhor, M. S.; Key, J. A.; Strelkov, I. S.; Cairo, C. W. J. Org. Chem. 2009, 74, 8669; (b) Goretta, S. A.; Kinoshita, M.; Mori, S.; Tsuchikawa, H.; Matsumori, N.; Murata, M. Bioorg. Med. Chem. 2012, 20, 4012.

11. Cadiot, P.; Chodkiewicz, W. in Chemistry of Acetylenes; Viehe, H. G., Ed.; Marcel Dekker, New York, 1969; pp 597-647.

12. Ando, J.; Kinoshita, M.; Cui, J.; Yamakoshi, H.; Dodo, K.; Fujita, K.; Murata, M.; Sodeoka, M. Proc. Natl. Acad. Sci. U.S.A. 2015, 112,4558

13. Yin, W.; He, C.; Chen, M.; Zhang, H.; Lei, A. Org. Lett. 2009, 11, 709.

14. Terao, J.; Todo, H.; Begum, S. A.; Kuniyasu, H.; Kambe, N. Angew. Chem., Int. Ed. 2007, 46, 2086.

15. Matsumori, N.; Yasuda, T.; Okazaki, H.; Suzuki, T.; Yamaguchi, T.; Tsuchikawa, H.; Doi, M.; Oishi, T.; Murata, M. Biochemistry 2012, 51, 8363.

16. (a) Lethu, S.; Matsuoka, S.; Murata, M. Org. Lett. 2014, 16, 844. (b) Cui, J.; Lethu, S.; Yasuda, T.; Matsuoka, S.; Matsumori, N.; Sato, F.; Murata, M. Bioorg. Med. Chem. Lett. 2015, 2, 203.

17. See Supplementary data for details.

18. (a) Bunow, M. R.; Levin, I. W. Biochim. Biophys. Acta 1977, 489, 191; (b) Kabisch, G.; Möbius, G. Spectrochim. Acta Part A 1982, 38, 1195

19. Chol- $d$ was synthesized from commercial 5-cholesten-3-on by reduction using deuterated reducing reagent $\mathrm{NaBD}_{4}$. See Supplementary data for details.

20. (a) Guo, W.; Kurze, V.; Huber, T.; Afdhal, N. H.; Beyer, K.; Hamilton, J. A. Biophys. J. 2002, 83, 1465; (b) Murari, R.; Murari, M. P.; Baumann, W. J. Biochemistry 1986, 25, 1062.

21. Brzustowicz, M. R.; Cherezov, V.; Caffrey, M.; Stillwell, W.; Wassall, S. R. Biophys. J. 2002, 82, 285.

22. Oldfield, E.; Meadows, M.; Rice, D.; Jacobs, R. Biochemistry 1978, 17, 2727.

23. Matsumori, N.;Kasai, Y.; Oishi, T.; Murata, M.; Nomura, K. J. Am. Chem. Soc. 2008, 130, 4757.

24. Mehnert, T.; Jacob, K.; Bittman, R.; Beyer, K. Biophys. J. 2006, 90, 939.

25. Calhoun, W. I.; Shipley, G. G. Biochim. Biophys. Acta 1979, 555, 436.

26. Bartels, T.; Lankalapalli, R. S.; Bittman, R.; Beyer, K.; Brown, M. F. J. Am. Chem. Soc. 2008, 130, 14521.

27. Davis, J. H.; Jeffrey, K. R.; Bloom, M.; Valic, M. I.; Higgs, T. P. Chem. Phys. Lett. 1976, 42, 390. 


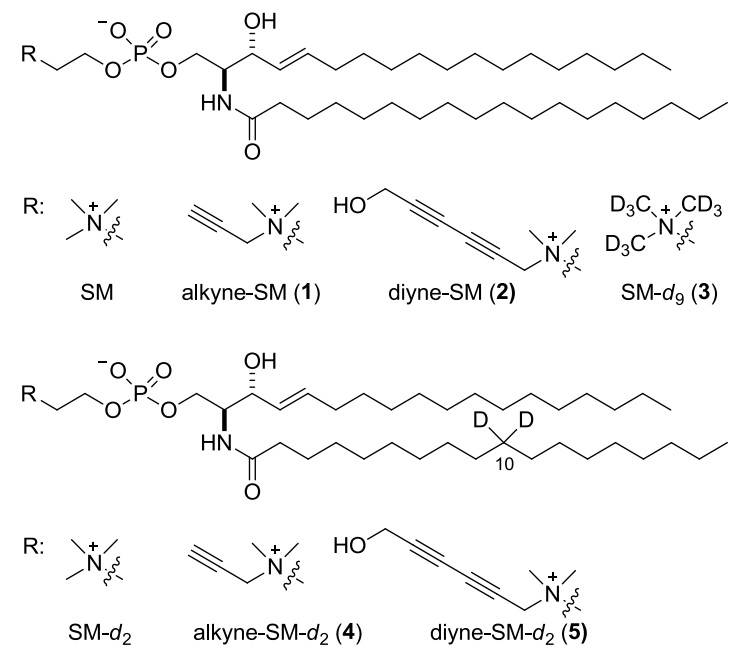

Figure 1. Chemical structures of natural SM, three Raman-tagged SMs and deuterated SMs discussed herein.

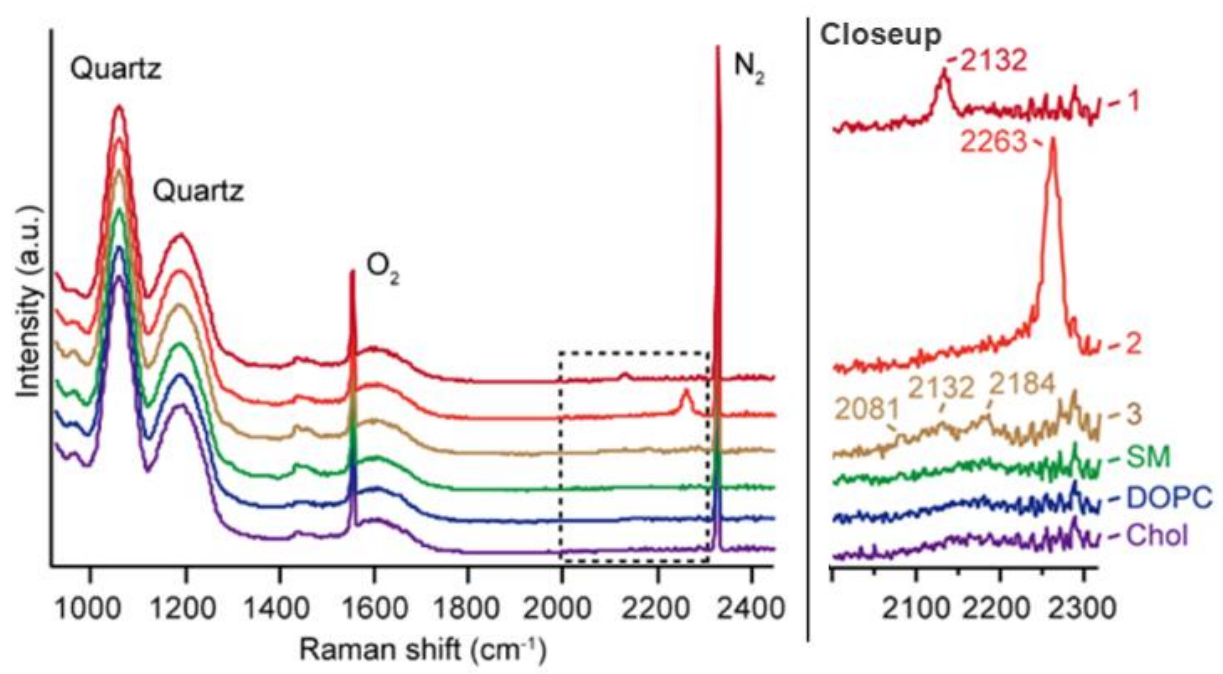

Figure 2. Raman spectra of pure alkyne-SM 1, diyne-SM 2, SM- $d_{9}$ 3, SM, DOPC and chol of quartz supported monolayers. Exposure time was $6 \mathrm{~s}$. Raman measurements were performed for 15 times per sample. Averaged Raman spectra are displayed in the image.

(a)<smiles>CCCCCCCCCCCCCCCCCCCC</smiles>

(b)

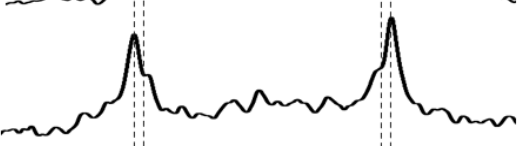

(c)

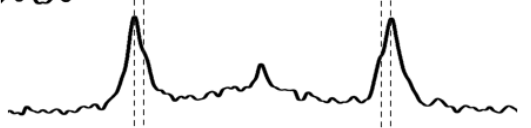

(d)

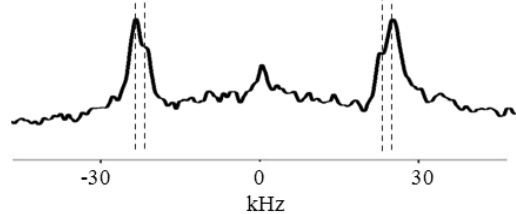

Figure 3. ${ }^{2} \mathrm{H}$ NMR spectra from oriented multi-bilayers of (a) chol- $d / \mathrm{DOPC}(1 / 1 \mathrm{~mol})$, (b) SM/chol- $d / \mathrm{DOPC}(1 / 1 / 1 \mathrm{~mol})$, (c) $\mathbf{1} / \mathrm{chol}-d / \mathrm{DOPC}$ $(1 / 1 / 1 \mathrm{~mol})$ and $(\mathrm{d}) 2 / \mathrm{chol}-d / \mathrm{DOPC}(1 / 1 / 1 \mathrm{~mol})$ at $25^{\circ} \mathrm{C}$. The angle of the membrane on glass supports was placed in parallel with the magnetic field $B_{\mathrm{o}}$ 


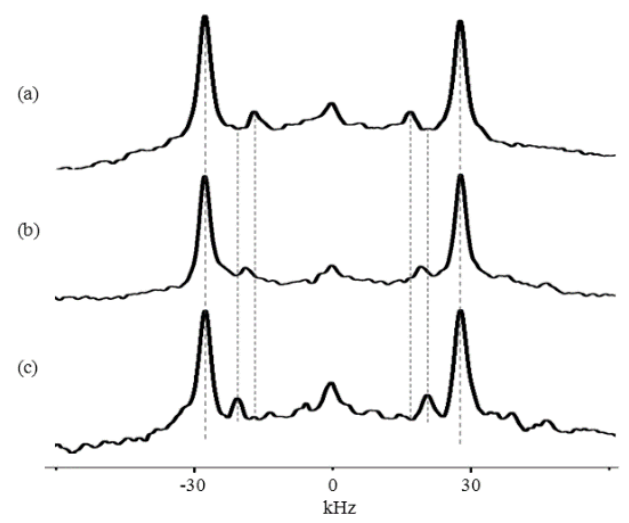

Figure 4. ${ }^{2} \mathrm{H}$ NMR spectra from oriented multi-bilayers of (a) SM- $d_{2} / \mathrm{DOPC} / \mathrm{chol}(1 / 1 / 1 \mathrm{~mol})$, (b) 4/DOPC/chol (1/1/1 mol) and (c) 5/DOPC/chol $(1 / 1 / 1 \mathrm{~mol})$ at $25^{\circ} \mathrm{C}$. The angle of the membrane on glass supports was placed in parallel with the magnetic field $B_{0}$.
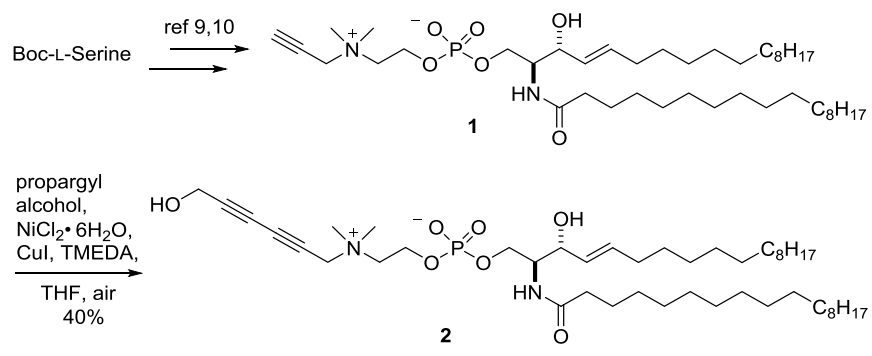

Scheme 1. Synthesis of alkyne-SM 1 and diyne-SM 2.

Boc-L-Serine $\stackrel{\text { ref } 9}{\longrightarrow}$
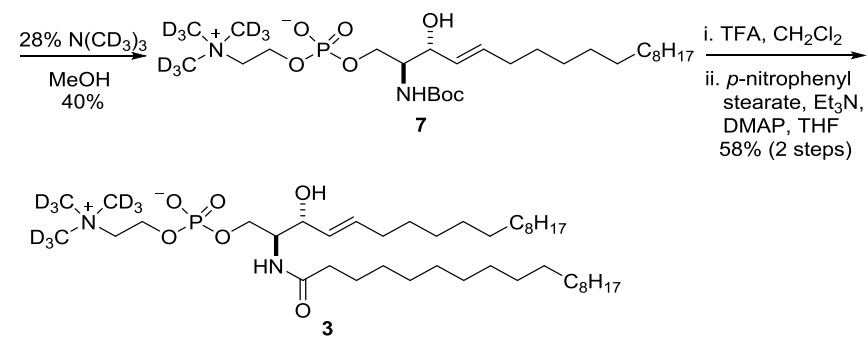

Scheme 2. Synthesis of SM- $d_{9} 3$ 


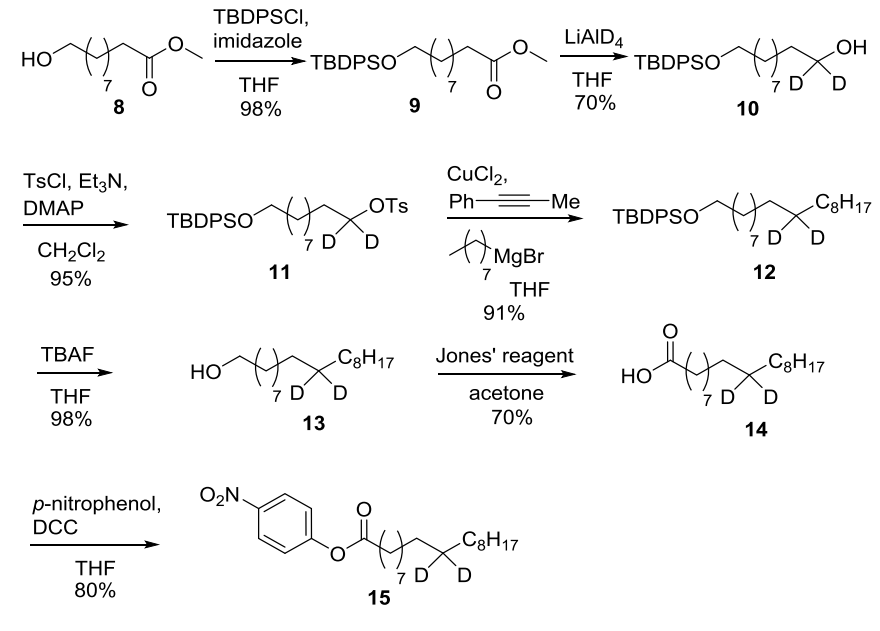

Scheme 3. Synthesis of deuterated stearic acid ester $\mathbf{1 5}$.

Table 1

Molecular ordering of chol- $d$ in SM, 1 and $\mathbf{2}$ membranes determined by ${ }^{2} \mathrm{H}$ NMR at $25^{\circ} \mathrm{C}$

\begin{tabular}{lccc}
\hline & $\begin{array}{c}\text { SM/chol-d/DOPC } \\
(1 / 1 / 1 \mathrm{~mol})\end{array}$ & $\begin{array}{c}\mathbf{1} / \text { chol-d/DOPC } \\
(1 / 1 / 1 \mathrm{~mol})\end{array}$ & $\begin{array}{c}\mathbf{2} / \text { chol-d/DOPC } \\
(1 / 1 / 1 \mathrm{~mol})\end{array}$ \\
\hline$\Delta v_{\mathrm{Q}}{ }^{\mathrm{a}}\left(\mathrm{L}_{\mathrm{o}}\right)$ & $48.2 \mathrm{kHz}$ & $47.7 \mathrm{kHz}$ & $47.8 \mathrm{kHz}$ \\
$\Delta v_{\mathrm{Q}}\left(\mathrm{L}_{\mathrm{d}}\right)$ & $43.1 \mathrm{kHz}$ & $43.9 \mathrm{kHz}$ & $43.7 \mathrm{kHz}$ \\
$S_{\mathrm{CD}}{ }^{\mathrm{b}}\left(\mathrm{L}_{\mathrm{o}}\right)$ & 0.38 & 0.38 & 0.38 \\
$S_{\mathrm{CD}}\left(\mathrm{L}_{\mathrm{d}}\right)$ & 0.34 & 0.35 & 0.35 \\
\hline
\end{tabular}

${ }^{\text {a }}$ Quadrupolar splitting.

${ }^{\mathrm{b}}$ Order parameter, describing the motion of the C-D bond with respect to the bilayer normal. This can be calculated via the following equation: ${ }^{22}$ $\Delta v_{\mathrm{Q}}=(3 / 4)\left(e^{2} q Q / h\right)\left|S_{\mathrm{CD}}\right|$, where $e^{2} q Q / h=168 \mathrm{kHz}$ is the static quadrupolar coupling constant.

Table 2

${ }^{2} \mathrm{H}$ NMR data of SM- $d_{2}, 4$ and 5 in membranes at $25^{\circ} \mathrm{C}$.

\begin{tabular}{lccc}
\hline & $\begin{array}{c}\mathrm{SM}-d_{2} / \mathrm{DOPC} / \mathrm{chol} \\
(1 / 1 / 1 \mathrm{~mol})\end{array}$ & $\begin{array}{c}\text { 4/DOPC/chol } \\
(1 / 1 / 1 \mathrm{~mol})\end{array}$ & $\begin{array}{c}5 / \mathrm{DOPC} / \mathrm{chol} \\
(1 / 1 / 1 \mathrm{~mol})\end{array}$ \\
\hline$\Delta v_{\mathrm{Q}}{ }^{\mathrm{a}}\left(\mathrm{L}_{\mathrm{o}}\right)$ & $55.3 \mathrm{kHz}$ & $55.2 \mathrm{kHz}$ & $55.4 \mathrm{kHz}$ \\
$\Delta v_{\mathrm{Q}}\left(\mathrm{L}_{\mathrm{d}}\right)$ & $34.1 \mathrm{kHz}$ & $38.3 \mathrm{kHz}$ & $41.0 \mathrm{kHz}$ \\
$S_{\mathrm{CD}}{ }^{\mathrm{b}}\left(\mathrm{L}_{\mathrm{o}}\right)$ & 0.44 & 0.44 & 0.44 \\
$S_{\mathrm{CD}}\left(\mathrm{L}_{\mathrm{d}}\right)$ & 0.27 & 0.30 & 0.33 \\
\hline
\end{tabular}

${ }^{\mathrm{a}}$ Quadrupolar splitting.

${ }^{\mathrm{b}}$ Order parameter, describing the motion of the C-D bond with respect to the bilayer normal. This can be calculated via the following equation:22 $\Delta v_{\mathrm{Q}}=(3 / 4)\left(e^{2} q Q / h\right)\left|S_{\mathrm{CD}}\right|$, where $e^{2} q Q / h=168 \mathrm{kHz}$ is the static quadrupolar coupling constant. 


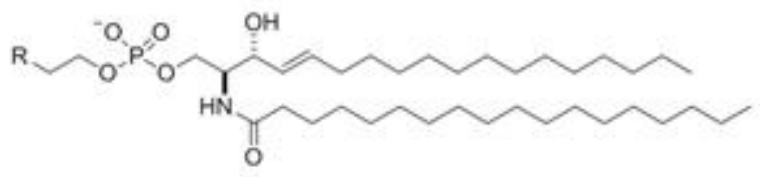

$\mathrm{R}$ :

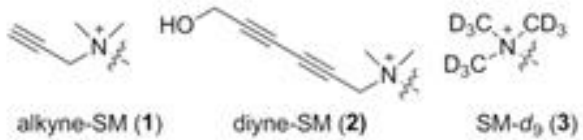

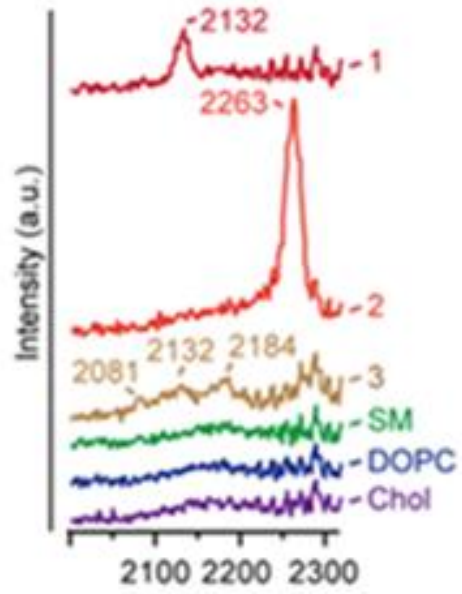

Raman shift (cm') 